\title{
Self-assembly of plasmonic nanostructures into superlattices for surface-enhanced Raman scattering applications
}

\author{
Rui Liu*, Shasha Li, Jing-Fu Liu \\ State Key Laboratory of Environmental Chemistry and Ecotoxicology, Research Center for Eco-Environmental Sciences, Chinese Academy of Sciences, Beijing \\ 100085, China
}

\section{A R T I C L E I N F O}

\section{Article history:}

Available online 20 September 2017

\section{Keywords:}

Surface enhanced Raman scattering

Self-assemble

Plasmonic superlattice

Ultrasensitive analysis

In-situ surface events monitoring

In-vivo biochemistry process study

\begin{abstract}
A B S T R A C T
Since the initial discovery that the interparticle nanogaps in nanoparticle aggregates was the surfaceenhanced Raman scattering (SERS) hotspots that facilitated the enormous enhancements in SERS, the controllable assembly of plasmonic nanoparticles into superstructures is recognized as the most effective strategy for fabricating SERS substrate. The homogenous distribution abundant SERS hotspots inside the self-assembly reproducibly amplify the SERS signal. This ultimately makes SERS an ultrasensitive analytical method with the potential to elicit fundamental chemistry, biology, and physics breakthroughs.

This review focuses on recent developments in self-assemble of plasmonic NPs for SERS applications, including:

1) Template-guided self-assemble of plasmonic NPs

2) Templateless self-assemble of plasmonic NPs

3) Taking full advantage of the assembly process: integration of target analyte recognition and capture capacity into the assembly

4) Assembly makes perfect: new opportunities created by large self-assembling SERS substrates.

5) Future directions and possible applications of self-assembled SERS substrates.
\end{abstract}

() 2017 Elsevier B.V. All rights reserved.

\section{Introduction}

Since the first report in 1970s on the significant enhanced Raman scattering on a metal substrate surface, i.e. surfaceenhanced Raman scattering (SERS) [1-3], SERS has continued to capture the attention of the scientific world. The material basis for this phenomenon has been primarily attributed to an intensified electromagnetic (EM) field, which is stimulated through the collective oscillation of free electrons in the metallic substrate and further enhanced through chemical means [4]. The research enthusiasm for SERS was further fueled by the demonstration of its genius in the provision of finger-print spectra at a single molecular (SM-SERS) sensitivity in 1997 [5,6]. Since then, its unparalleled sensitivity and molecular specificity resulted in SERS technology being listed as one of the most flourishing research areas in nanoscience and playing an increasing role in environmental protection [7], material characterization [8], in situ or operando

\footnotetext{
* Corresponding author.

E-mail address: ruiliu@rcees.ac.cn (R. Liu).
}

physicochemical process studies [9], and in vivo studies of biological pathways and supra-molecular dynamics in living cells [10].

However, the previously described enhancements, which were determined to be $10^{8}$ and $10^{10-11}$ for SM-SERS for resonant and non-resonant probe molecules [11], respectively, have been unattainable with separated Au and Ag NPs neither. Both theoretical predictions and experimental results have revealed the SERS enhancement factor (EF) for most Ag and Au NPs is within the range of $10^{3}$ to $10^{4}$ [12]. Even with shape/size optimization and judicious selection of the excitation photon energy to resonance mode, the EF was still in the order of $10^{5}$ [13]. In fact, in the initial report on SMSERS, the Ag colloid was "activated" by low concentrations of $\mathrm{NaCl}$ [5]. As revealed by correlating the SERS and dark-field optical scattering spectra, the NPs that had themselves given rise to the SM-SERS spectra had red-shifted spectra with multiple peaks (Fig. 1A) [4,5], which stemmed from the strong interparticle coupling of the EM field inside the nanogaps between NP pairs, clusters, and aggregate films. This was further substantiated by the exponential increase in average ensemble SERS EF for NP clusters with a well-defined geometry (Fig. 1B and C) [12,14], and the direct 


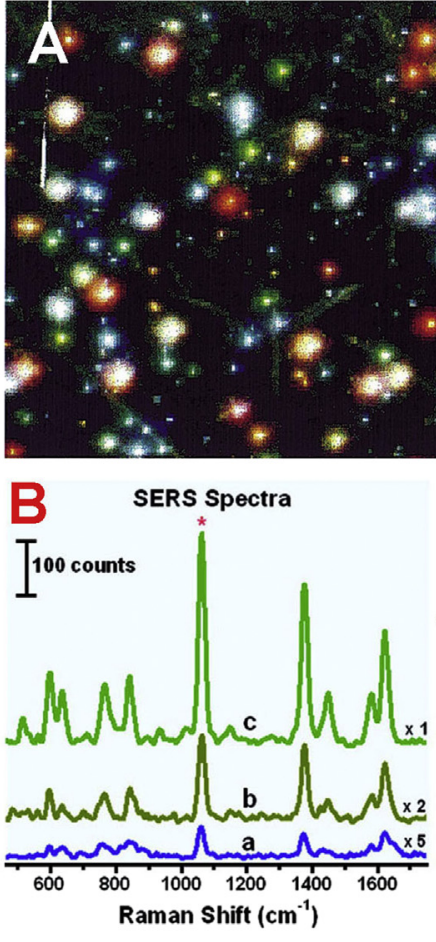

Ensemble-Averaged SERS Intensity ratio:

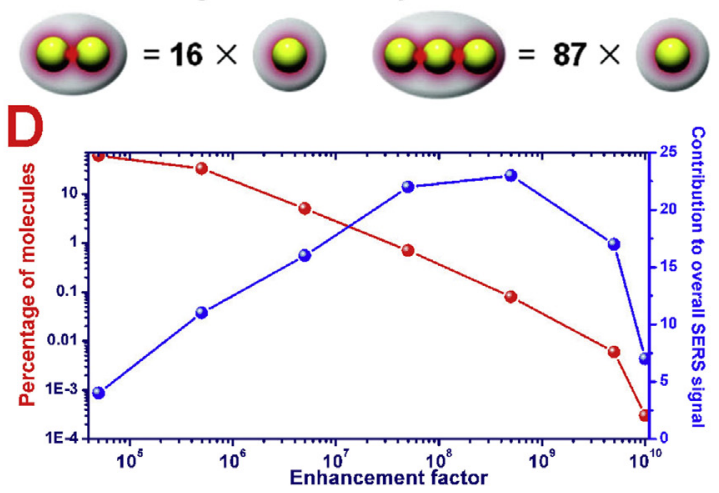

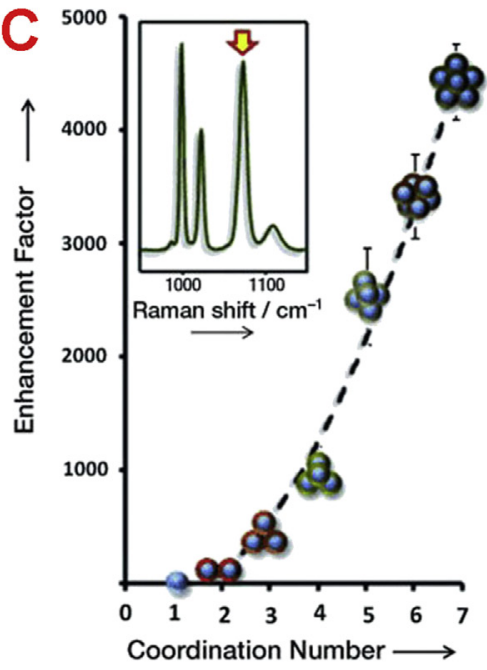
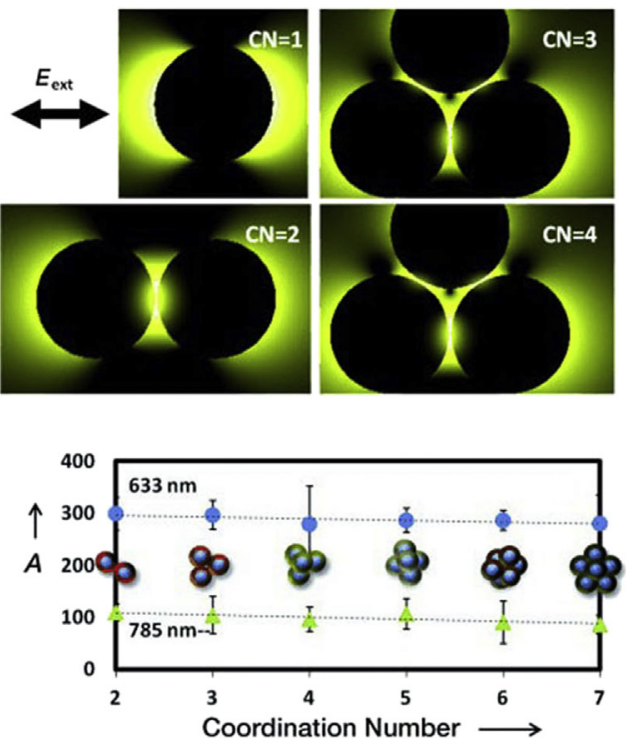

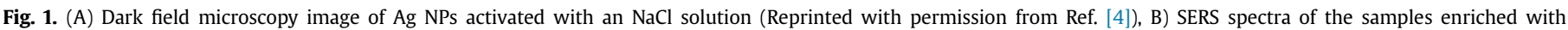

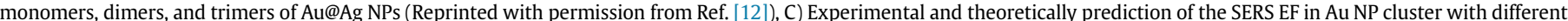
coordination number and geometry (Reprinted with permission from Ref. [14]), and the distribution of SERS EF in silver-coated nanosphere lattice (data from Ref. [17]).

observation of a $10^{8}$ enhancement of the molecules inside SERS hotspots consisting of $\mathrm{Ag}$ nanocubes after removal of the molecule outside the hotspots using plasma etching [15]. Unfortunately, due to the low structural uniformity when NP aggregation is induced by salt, colloidal silver has a remarkably heterogeneous distribution of spectral properties, suggesting the uneven distribution of SERS hotspots [16]. Moreover, further work has demonstrated SERS hotspots with EFs higher than $10^{8}$ and $10^{10}$ account for less than 0.09 and $0.0003 \%$, respectively, of the total sites taken up by probe molecules (Fig. 1D) [17]. The insufficiency of SERS hotspots and their uneven distribution are the primary factors hindering the development of SERS-based methods for quantitative analytic applications, and therefore extensive research has been done in designing hierarchical plasmonic architectures that are advantageous in SERS substrates.

To this end, top-down strategies, such as electron beam lithographic and ion beam etching, have been employed to fabricate various nanostructures with built-in nanogaps as SERS hotspots $[18,19]$. However, in addition to their disadvantages of being time- and labor-consuming, the resolution of these processes is limited to $\sim 10 \mathrm{~nm}$ and the presence of rough surfaces and multiple crystalline domains further decreases control over SERS performance [20]. On the other hand, through stimulation with hydrophobic/solvophobic forces, surface intension, Van der Waals and electrostatic attraction forces, hydrogen bonds, and covalent interactions, NPs can be triggered in solution or at solution interfaces to self-assemble into either small clusters or wafer scale superlattices, which are comprised of thousands of NPs with reduced interparticle distances of 1-2 nm (Fig. 2) [14,21-28]. Furthermore, the self-assembly process enables the creation of nanoarchitectures with specific optical resonances and induces the generation of relatively uniformly distributed hotspots, which are challenging or even impossible to create with conventional top-down technologies. Meanwhile, by changing the number/shape of the NPs in the assembly, varying the directionality of NP organization, and finetuning interparticle distance, the optical properties and, therefore, the distribution of the generated EM can be notably manipulated. Moreover, from an analytical chemistry viewpoint, the self- 

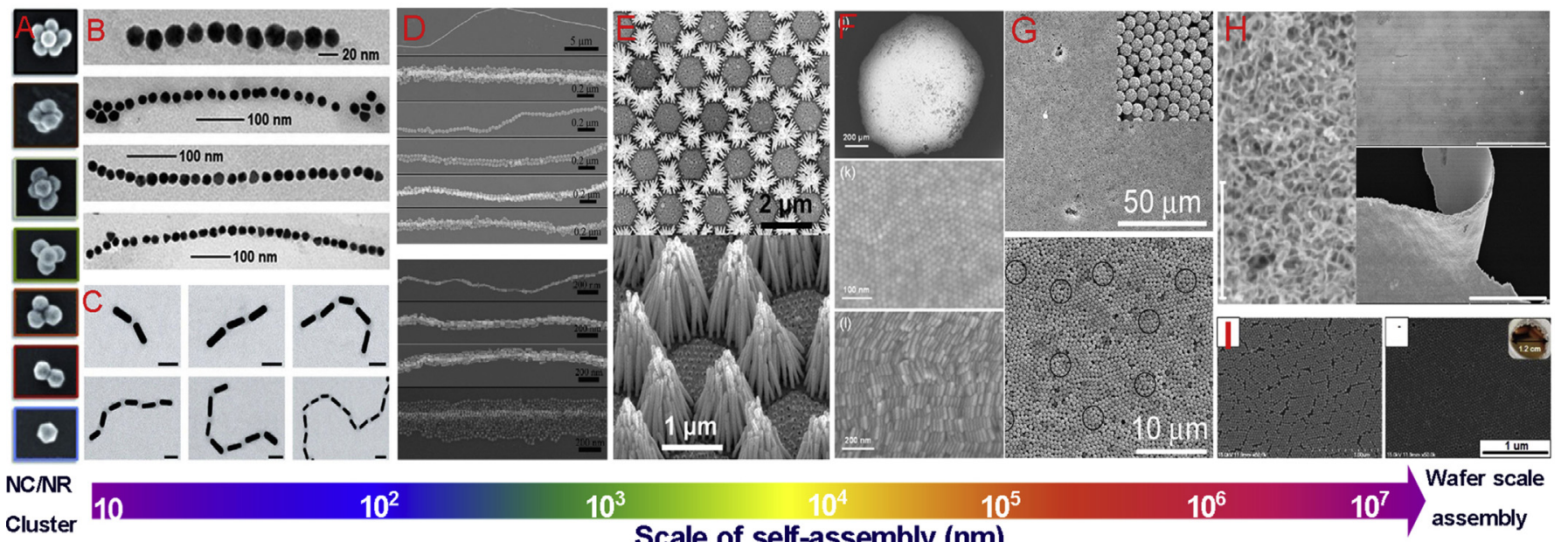

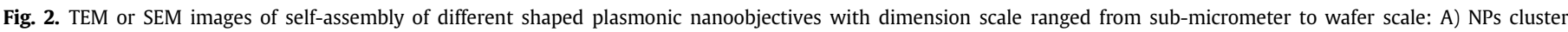

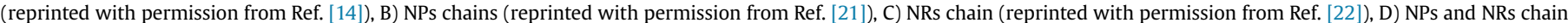

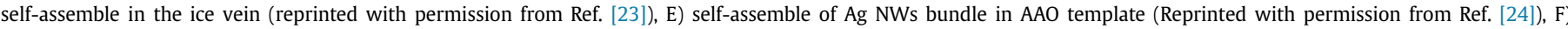

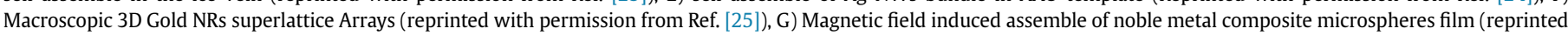

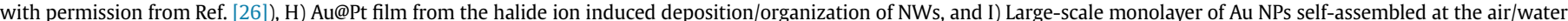
interface (reprinted with permission from Ref. [28])

assembly process is facile for coupling of molecular recognition [29-31] and target analyte capture/enrichment capacity into the SERS substrate [7,32], as well as integrating multiple functionality components together. This significantly increases the range of fields in which SERS is applicable (Table 1) [33,34].

In this present article, we review recent progress on the selfassembly of plasmonic nanostructures as SERS substrates. After briefly discussing the importance of plasmonic self-assembly for SERS analysis, we review experimental advances in the selfassembly of plasmonic nanostructures guided by templates, induced by nano-forces, and stimulated through external fields. The next section proposes how to take full advantage of this assembly from an analytical chemistry angle and is followed by a discussion of the new opportunities created by self-assembly. Finally, we summarize the future directions of research in the field of self-assembling SERS substrates.

\section{Template-guided self-assembly}

\subsection{Zero-dimensional plasmonic cluster}

A template is defined in this article as organic molecules that, when present in a large group, are capable of bringing NPs together and assembling them into clusters with different coordination numbers via covalent bonds or electrostatic interactions. For example, a classic method of creating NP dimers and trimers is interconnecting NPs with molecular linkers, such as thiolated nucleic acids and long-chain organic molecules, and sorting the resulting cluster by sedimentation, electrophoresis, or density gradient centrifugation [35]. The carbodiimide chemistry between amino- and carboxylic-group functionalized NPs also enables the generation of NP dimers. Another effective strategy for guiding NP assembly into clusters involves the electronic interactions between charged NPs and polyelectrolytes with the opposing charge [36].

Compared with NP ensembles with more complex structures, NP clusters are a more ideal platform on which to study how structural parameters, especially the number of and the distance between interparticle gaps, influence SERS performance. This can be done both experimentally by determining the average EF of the ensemble and theoretically by solving the Maxwell equations, e.g. finite-difference time-domain simulation. For example, by using Raman probe-loaded Au@Ag dimers and trimers created by $\mathrm{NaCl}$ induced linear aggregation of NPs by polystyrene-blockpoly(acrylic acid) (PSPAA) encapsulation and density gradient centrifugation, it was found that the relative SERS intensities from dimers and trimers were 16- and 87-fold higher, respectively, than

Table 1

Comparison of the different method for SERS substrate fabrication.

\begin{tabular}{|c|c|c|c|c|c|c|c|}
\hline & $\begin{array}{l}\text { Scale of } \\
\text { assemble }\end{array}$ & $\begin{array}{l}\text { Size of } \\
\text { nanogaps }\end{array}$ & Uniformity & $\begin{array}{l}\text { Speed of } \\
\text { method }\end{array}$ & $\begin{array}{l}\text { Controllability } \\
\text { over morphology }\end{array}$ & $\begin{array}{l}\text { Easy to } \\
\text { operation }\end{array}$ & $\begin{array}{l}\text { SERS } \\
\text { performance }\end{array}$ \\
\hline $\begin{array}{l}\text { Electron beam } \\
\text { lithographic }\end{array}$ & $<10^{3} \mathrm{~nm}$ & $10 \mathrm{~nm}$ or above & High & Slow & High & No & Moderate \\
\hline Ion beam etching & $<10^{3} \mathrm{~nm}$ & $10 \mathrm{~nm}$ or above & High & Slow & High & No & Moderate \\
\hline Dealloying & Up to $10^{7} \mathrm{~nm}$ & $10 \mathrm{~nm}$ or above & High & Fast & Low & Yes & Moderate \\
\hline $\begin{array}{l}\text { Templateless } \\
\text { assemble }\end{array}$ & $10^{2} \sim 10^{7} \mathrm{~nm}$ & Down to $1-2 \mathrm{~nm}$ & Low or high & Fast & Low to high & $\begin{array}{l}\text { Driving force } \\
\text { Dependent }\end{array}$ & $\begin{array}{l}\text { Moderate } \\
\text { to Good }\end{array}$ \\
\hline $\begin{array}{l}\text { Template-guided } \\
\text { assemble }\end{array}$ & $10^{2} \sim 10^{7} \mathrm{~nm}$ & Down to $1-2 \mathrm{~nm}$ & High & Fast & Low to high & $\begin{array}{l}\text { Template } \\
\text { dependent }\end{array}$ & $\begin{array}{l}\text { Moderate } \\
\text { to Good }\end{array}$ \\
\hline $\begin{array}{l}\text { Halide ion } \\
\text { induced assemble } \\
\text { of nanowires }\end{array}$ & Wafer scale & Down to $1-2 \mathrm{~nm}$ & High & Fast & High & Yes & excellent \\
\hline $\begin{array}{l}\text { Gelation of } \\
\text { nanoparticles }\end{array}$ & Up to $10^{7} \mathrm{~nm}$ & $10 \mathrm{~nm}$ or above & Low & Very slow & Moderate & No & Good \\
\hline
\end{tabular}


monomers [12]. An elegant study in this field was published by Pazos-Perez et al., where block copolymers of poly(ethylene oxide)b-poly(propylene oxide)-b-poly(ethylene oxide) (Pluronic F-68, PF68) were used as "glue" to produce very symmetrical gold NP clusters with different coordination numbers [14]. The formed clusters were separated into dimers, trimers, tetramers, and a complex mixture of trigonal bipyramids, octahedrals, and pentagonal bipyramids with CNs ranging from 2 to 7, respectively. By clearly separating the synthesized clusters with well-defined structures, the relationship between optical properties and, thus, MF field enhancement and the structure of the NP clusters were clearly deciphered. Both model fitting and experimental results demonstrated as $\mathrm{CN}$ increased from 1 (one single NP) to 7 (pentagonal bipyramids), the SERS EF increased by 4000 -fold for multiple couplings of EM fields.

In addition to serving as a model structure for deciphering the structure-SERS activity relationship, zero-dimension plasmonic assemblies have also demonstrated the importance of analytical chemistry, especially when analyzing bio-macromolecules [36-38]. The electrostatic adhesion between the phosphate backbones in DNA and RNA promotes the aggregation of negatively charged silver NPs into stable clusters. Through this, DNA strands are precisely packed into the nanogaps, resulting in an intense and reproducible enhancement of SERS. Also, aside from inducing controllable aggregation of NPs, the phosphate backbone signal is an ideal internal standard for calibrating the absolute signal of each base and enables reliable characterization of the DNA structure [37]. Due to the significant improvements in sensitivity and accuracy, very small amounts of DNA (approximately the amount extracted from 10 to 100 cells) was sufficient for direct SERS analysis without amplification, thus providing accurate and direct information on DNA in its native state. Taking advantage of this strategy, quantitative recognition of hybridization events, as well as the detection of single base mismatches and base methylations in duplexes, was achieved [36].

\subsection{One-dimensional (1D) plasmonic assembly}

One-dimensional materials, such as nanowires, nanotubes and their array, are also widely used as hard templates to guide/confine the assembly of plasmonic NPs. Driven by capillary forces, Au NPs can be internalized and self-assembled into subnanometerseparated peapod-like linear NP chains inside silica nanotubes (SNTs) using a repeated wet-dry procedure [39]. Interestingly, the geometric configurations of the Au NPs inside the SNTs, as well as the chain lengths, were tunable between single- and double-lines by controlling the diameters of the Au NPs and the orifice of the SNTs. Moreover, the SNTs preserved the structural characteristics of the nanochains and the SERS signal, which ultimately lead to the design of a SERS-based intercellular pH sensor that was very sensitive and stable.

Unlike hard templates that direct the self-assembly of nanochains using physical confinement, soft templates, such as Langmuir monolayers and linear polymer conjugates, are also very effective at guiding the 1D self-assembly of NPs, e.g. by covalent forces $[40,41]$. In the presence of the long-chain ionic liquid 1hexadecyl-3-methylimidazolium bromide $\left(\mathrm{C}_{16} \mathrm{mimBr}\right)$ Langmuir monolayer, Gold NPs were synthesized in one step through the reduction of $\mathrm{AuCl}_{4}^{-}$ions by UV-light irradiation and self-assembly into nanochains at the air/water interface [40]. The assembly process was driven by $\pi-\pi$ interactions between the imidazole rings and steric hindrance of $\mathrm{C}_{16} \mathrm{mimBr}$ suppressed the formation of nanocrystals with large areas. Concurrently, the structures and, therefore, the optical/SERS properties of the nanochains were easily manipulated by changing the reaction temperature [40]. Another example involves the use of hyaluronic acid (HA) and hydrocaffeic acid (HCA) conjugates to guide the self-assembly of citratestabilized gold NPs into nanochains. Here, HCA serves as a highaffinity anchor for Au NPs and facilitates their linear assembly on linear nonsulfated glycosaminoglycan HA [41]. Due to strong interparticle coupling, the assembled Au NPs chains exhibit maximal absorption in the near-infrared region and satisfactory SERS performance. After being functionalized with photosensitizers (PS), more than 99\% of Au NPs nanochains can be internalized by cells and there is notably enhanced phototoxicity over PS alone, making these very promising for applications in theranostics, where SERS imaging and photodynamic therapies would be integrated [41].

Besides physical templates with fixed morphologies, any substrate with confined spaces, e.g. veins formed from frozen water, can effectively guide the 1-D assembly of Au and Ag NPs [23,42]. Freezing of an aqueous Au NP colloid results in exclusion of NPs from ice grains as impurities, and chains with lengths of up to micrometer-scale were formed in the ice veins. It is important to note that the confining effects of the nanoscale ice veins at the final stage of freezing strongly influenced the arrangement of the NPs, which is very important for the assembly of anisotropic nanostructures. For example, Au nanorods (NRs) follow an end-to-end rather than side-by-side conformation in the formed nanochains [23]. Combining field emission scanning electron microscope (FESEM) and SERS mapping, our group demonstrated that the nanochains formed in ice-veins are effective SERS substrates with superior EFs and reproducibility over SERS substrates formed dry at room temperature with an equal amount of Au NPs. This enabled us to detect trace Thiram on the surface of an apple with a sensitivity as low as $0.28 \mathrm{ng} / \mathrm{cm}^{2}[42]$.

\subsection{Two-dimensional plasmonic assembly}

Two-dimensional (2D) materials, including graphene $(\mathrm{G})$, graphene oxide (GO) [32,43-46], $\mathrm{MoS}_{2},[16,47]$ and hexagonal boron nitride [48], are good scaffolds for the self-assembly of NPs to become SERS substrates. In addition to the rich surface functional groups that can be used as anchoring sites at which to fix Au and $\mathrm{Ag}$ NPs, GO has a high adsorption capacity for target analytes [49], such as environmental pollutants and biological molecules, which further enhances SERS analysis of $\mathrm{Au}$ and $\mathrm{Ag}$ assemblies on GO $[32,44]$. Moreover, the atomic-layer thickness, distinctive electronic structure, and flexibility of G and GO are advantages not available from other materials, offering an alternative route for chemical enhancement. This was principally demonstrated by the 20 -fold enhancement of the Raman signal of dye molecules adsorbed on $\mathrm{G}$ monolayer [45]. This work was further expanded to combine the SERS performance of Au/Ag NPs (EM enhancement) and G monolayer (chemical enhancement). Here, a $G$ monolayer was created by transfer chemical vapor deposition on the surface of an $\mathrm{Au} / \mathrm{Ag}$ layer, where the surface tension and strong interactions between $\mathrm{Au} / \mathrm{Ag}$ and $\mathrm{G}$ enabled the self-assembly of $\mathrm{Au} / \mathrm{Ag}$ atoms into nanoislands with separated nanogaps [46]. The benefits of this include physical isolation of probe molecules from adsorption on the metal surface, clean vibrational information free from various metal-molecule interactions, and photo-stable SERS spectra. After being supported on polymer, this freestanding, transparent, and flexible " $G$ SERS tape" was very effective at direct, real-time, and reliable detection of trace amounts of analytes with high universality and systems with various surfaces. To obtain good flexibility of $G$, the intergap distance between nanoislands can be significantly shortened using thermally induced shrinking of $G$, which will further increase the SERS activity of G-SERS [50]. G was also utilized as a physical spacer to generate subnanometer gaps in Ag NP assemblies 
and Ag films to achieve multiple couplings between NPs and NPs/ film. This G-embodied structure had 1700-fold higher SERS EF than $\mathrm{Ag}$ NPs alone [51]. Meanwhile, to enhance the chemical stability of SERS-active Ag nanostructures, silver nanoplates were sandwiched between GO assembly layer-by-layer films into $\mathrm{rGO} / \mathrm{Ag} / \mathrm{rGO}$ nanomembranes, which transfer conformable coatings to complex surfaces and support a cleaner Raman signature [52].

Amongst the reported works on the self-assemble of $\mathrm{Au}$ or $\mathrm{Ag}$ NPs on 2D materials, Sun et al. did a lot of interesting jobs and designed a series of novel plasmonic nanostructures [53-55]. These nanostructures either referred to assemble of Ag NPs on the floral-clustered $\mathrm{G}$ nanosheets [53] and $\mathrm{MoS}_{2}$ monolayer [54], or Ag bowtie nanoantenna arrays on $\mathrm{G}$ with controllable layers [55]. Beside shown satisfactory SERS performance, the fabricated Ag assemble also displayed support material dependent behavior during utilizing the surface plasmon driven chemical reaction, which showing the perspective of SERS in the study of the interaction between metal NPs and 2D materials.

\subsection{Self-assembly on three-dimensional scaffolds}

While fabrication of 2D materials to serve as SERS substrates was hugely successful, optical scattering/light collection occurs in a three-dimensional (3D) focal volume. Therefore, the assembly of plasmonic NPs in a 3D pattern containing densely packed metal nanostructures with nanogaps to serve as SERS hotspots would be more conducive to maximizing SERS performance. For example, employing polystyrene spheres patterned with anodic aluminum oxide (AAO) as templates, and driven by capillary force, electrochemically deposited Ag NRs were, allowing to form hierarchical NR bundles with increased SERS activity for the ultrasensitive detection of pollutant with EFs as high as $10^{8}$, good uniformity, and high reproducibility of SERS signal (RSD <10\%) [24]. Meanwhile, based on the fact that $\mathrm{Au}$ atoms have different diffusion barriers, surface wettability, and nucleation rates on smooth carbon nanotubes (CNTs) and CNT/CNT intersections, 3D SERS-active Au assemblies in aligned carbon nanotubes were also synthesized [56].

\subsection{External field-induced self-assembly}

Self-assembly of plasmonic NPs also takes place due to externally applied magnetic and electric fields, and the applied field played a role that is similar with other templates that guide the assembly of NPs. After capturing analyte from aqueous samples, monodispersed $\mathrm{Fe}_{3} \mathrm{O}_{4} @ \mathrm{SiO}_{2} @ \mathrm{Ag}$ microspheres with hierarchical surfaces were collected using an external magnet and then magnetically self-assembled into uniform films [26]. Upon being air-dried on Si substrate, the assemblies formed large areas and stable films even once the applied field was removed. No "locking" ligands, such as polymer, were needed to prevent the assembly from disassembling. On the other hand, an applied voltage between 5 and $10 \mathrm{~V}$ has also been reported to drive self-assembly of Ag NPs into micrometer-sized chains across electrode gaps in air [57]. Of note, the reagent-free and, thus, interference-free nature of the magnetic/electro field-driven SERS substrate may have uses in ultrasensitive SERS analysis as SERS substrate, despite the relative sparseness of reports on applications of external field induced selfassembly.

\section{Templateless self-assembly}

\subsection{Interfacial assembly}

Despite the large surface energy of NPs, they are in a thermodynamically metastable state through temperate stabilization by electrostatic repulsion, steric hindrance, and solvation layer relevant mechanical repulsion associated with surface-adsorbed charged ions, surfactants, and solvent molecules, respectively. In the bulk phase of the NP colloid, the Brownian motion of the NPs inhibits their sedimentation and induces formation of a uniform solution. However, at the liquid-air or liquid-liquid interface, the strong surface tension and, therefore, the high Gibbs free energy recruits anything capable of lowering this energy, including NPs, to the interfacial region. For example, our group used R6G-labeled Ag NPs in situ SERS monitored their interface transfer process and found surface enrichment of Ag NPs. Further quantitative analysis by inductively coupled plasma mass spectrometry (ICP-MS) demonstrated that $\mathrm{Ag}$ in the surface layer had a concentration at least 100 fold higher than in the bulk phase [58]. However, this was not enough for the self-assembly of Ag NPs into practical SERS substrate, as no $\mathrm{Ag}$ film formed at the water/air interface and the majority of NPs remained in the bulk phase. From a dynamic viewpoint, there is a finite sorption barrier that needs to be conquered before these NPs can be moved to the interface zone. On the other hand, upon the enrichment of NPs, the notable increase in electronic repulsion between NPs as they approach one other makes such processes thermodynamically unfavorable. While the former can be overcome by shaking, heating, or evaporation of solvent, overcoming the latter is much more complicated and usually requires the addition of polymer, electrolyte, or surfactant to decrease the repulsion or increase the Van der Waals attraction forces.

\subsection{Liquid-liquid interface}

It is well-recognized that, when facilitated by surfactant (especially tetra-alkylammonium salt) and alkanethiols, NPs can be triggered at water-oil interfaces to self-assemble into thin films. This is attributed to the increased hydrophobicity of NPs to the attached chemicals. Using in situ SERS, it was observed that Au NP films form on the water-oil interface independent of the adsorption of added surfactant/alkanethiols on the surface (Fig. 3A) [59]. Based on this observation, a "modifier" and "promoter" mechanism was proposed to account for the assembly of NPs at the oil-water interface (Fig. 3B). Unlike alkanethiol molecules that adsorb on the NPs surface via strong Au-S bonds and drive Au NPs self-assembly with hydrophobicity, the added tetra-alkylammonium salt crosses the water/oil interface and promotes the interfacial assembly of NPs by shielding them from the electrostatic repulsion of the oil.

Besides the modifier/promoter-induced self-assembly of NPs at the oil-water interface, solvent that is soluble in both oil and water, such as ethanol, acetone, tetrahydrofuran, N,N-dimethylformamide, and isopropanol, is capable of inducing the self-assembly of nano-objectives with different morphologies (NPs, nanowires, nanosheets, or a combination of these) and different compositions ( $\mathrm{Au}, \mathrm{Ag}$, or graphene) at the oil-water interface. The added solvent may modulate the interfacial energy and polarity of the solution, as well as destroy the surface solvent layer on the nano-objective and decrease the colloid stability [60]. This solvent induced selfassembly strategy has been further employed to form a selfsupported Au NP film on a poly(methyl methacrylate) (PMMA) template, which was generated by the self-assembly of PMMA dissolved in toluene (Fig. 3C). This SERS substrate is flexible as a result of the flexibility of the PMMA film and is capable of in situ SERS detection of various target analytes on different matrixes, e.g. residual malachite green on the surface of fish [61]. This SERS substrate is very promising in the area of food safety due to its stability, ease of preparation, and applicability for in situ ultrasensitive SERS analysis on the surface of irregular objects.

Compared with their counterparts formed by the judicious selection of modifiers/promoters, solvent-induced self-assembled 

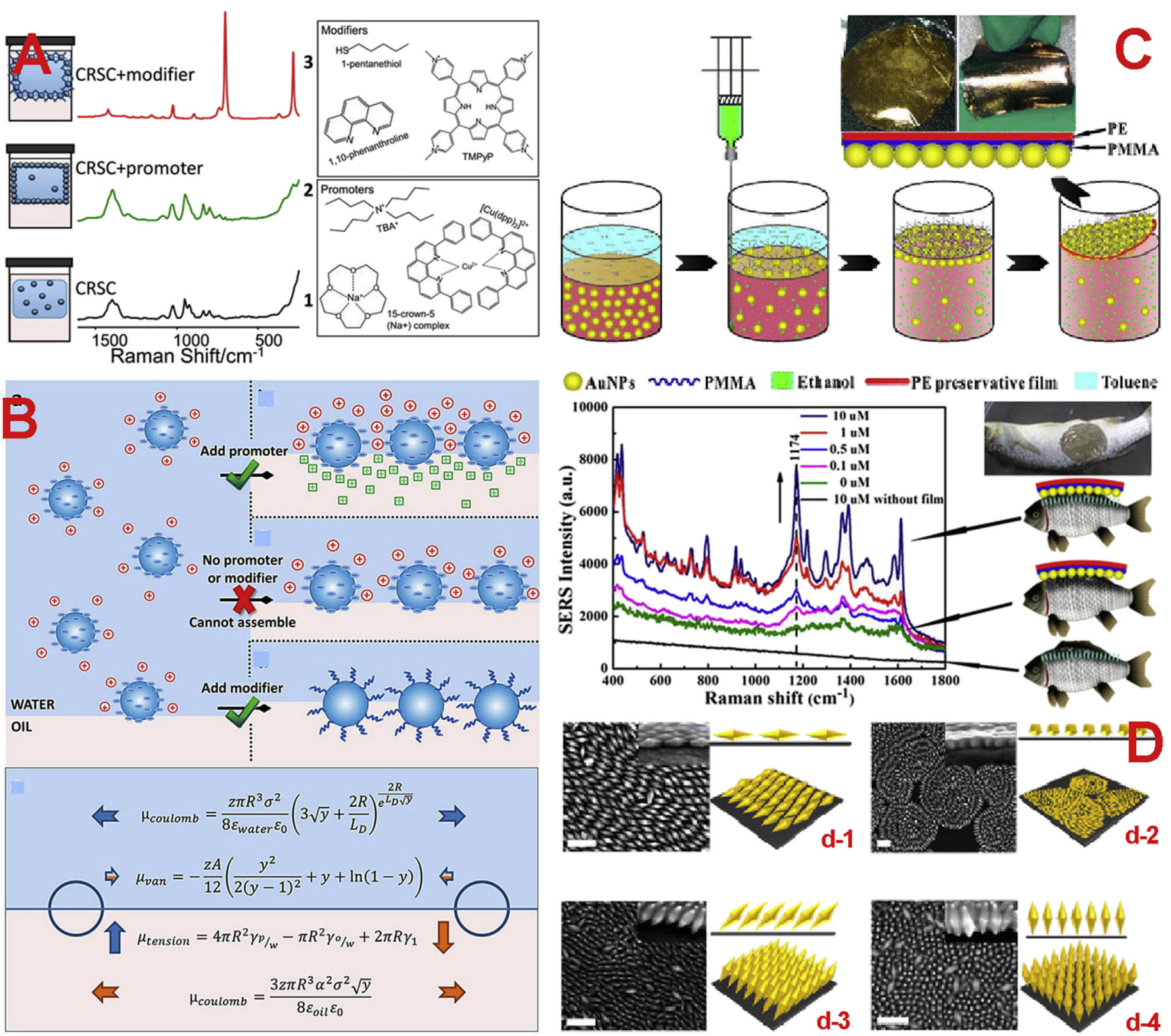

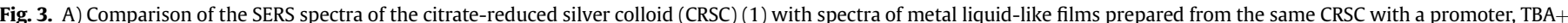

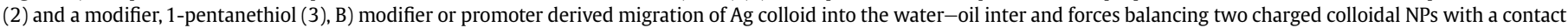

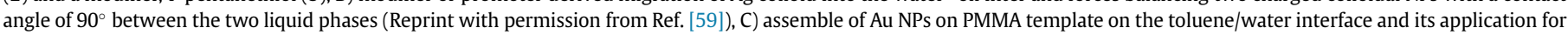

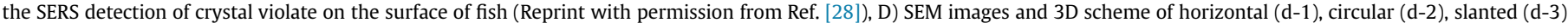
and vertical (d-4) alignment of bipyramid gold NPs a Si wafer (Reprint with permission from Ref. [67]).

film has a less ordered structure. However, this method displays superiority in material generality, as well as offers an unmodified metal surface for the adsorption of target analyte. Compared to other methods of SERS substrate fabrication, an important advantage of assembly at the oil-water interface is it enables the simultaneous formation of SERS substrate and the capture of target analyte. This means SERS detection can be easily coupled with solvent extraction, which largely suppresses the matrix effect and increases detection sensitivity. Taking advantage of this strategy, Ma et al. detected trace drug molecules in a urine sample [62].

In addition to the formation of a tightly packed 2D-nanostructure film in which Brownian movement is completely suppressed by Van der Waals interactions, Au NRs were reported to spontaneously undergo vertical alignment at the water-oil interface [63]. Theoretical stimulation indicates the interfacial energy can be lowered by $1500 \mathrm{k}_{\mathrm{B}} \mathrm{T}$, which largely exceeds that of thermal fluctuation (at $\mathrm{k}_{\mathrm{B}} \mathrm{T}$ level), through self-assembly into this configuration. This resulted in the collective coupling of the EM field, and SERS enhancement up to three to four orders of magnitude higher than from a solution of randomly oriented gold NRs.

\subsection{Liquid-air interface self-assembly of NPS into SERS substrate}

Compared with the liquid-liquid assembly associated with the addition of a water immiscible organic phase, which can contaminate the Au NP surface and occupy interstices, liquid-air is more simple and effective. Therefore, many interesting examples of NPs assembled at this region into SERS substrates have been reported $[28,43,58]$. Langmuir-Blodgett (LB) technology is the most effective and widely used strategy for the assembly of NPs at the air/ 
water interface $[64,65]$. This involves spreading organic solutions containing NPs with different morphologies, chemical compositions, and stabilized with different passivating agents at the air/ water interface, and then transferring the NP monolayers onto suitable substrates using the LB technique. Naturally, the assembled monolayers of plasmonic NPs, e.g. aligned silver nanowires, are excellent SERS substrates [66].

In addition to facilitating the formation of self-assembled LB monolayers using Langmuir film balance, Au NPs can also be selfassembled into monolayers at the liquid-air interface through modulation of the evaporation rate with a homemade chimney device. This is because, as the water evaporates, Au NPs are transported to the interface and effectively trapped by surface tension, forming a two-dimensional (2D) hexagonal close-packed (HCP) structure with interparticle gaps smaller than $2 \mathrm{~nm}$ [28]. The proper evaporation rate, the presence of surface-adsorbed hydroxylamine hydrochloride, and polyvinylpyrrolidone (PVP) were identified as the key factors behind the formation of the elegant $\mathrm{Au}$ NP monolayer. When the evaporation rate is too high, Au NPs aggregates also form at the interface. The presence of hydroxylamine hydrochloride on the Au surface renders these NPs positively charged. The presence of PVP, which is negatively charged at most pHs, allows partial replacement of the citrate ions on the NP surfaces and reduces the negative surface charges. Moreover, the nonpolar methylene and methine groups in the ring and backbone of PVP render the Au NPs weakly hydrophilic. Accordingly, the finite sorption barrier is reduced because of decreased interactions in the electric double layer. Meanwhile, the increased long-range particleinterface hydrophobic interaction between the Au NPs become dominant as the number of NPs at the interface increases and counter-balances the effect of steric repulsion. This stabilizes the monolayer film of NPs at the air/water interface [28]. The formed NP assembly can be easily transferred and can form at a wafer scale $\left(\mathrm{cm}^{2}\right.$ scale) in monolayer films on any substrate. When employed as a SERS substrate, the formed Au film not only generates a highly reproducible SERS signal with a large surface EF $\left(\sim 10^{6}\right)$ and high stability ( 45 days), but is also capable of working as a SERS active electrode for in situ SERS monitoring of electrochemical adsorption/ desorption.

As discussed in the first part of this review, the EM field and, therefore, the SERS performance of NP assemblies increase exponentially with decreases in interparticle distance. However, the presence of PVP molecules is necessary for the stability and assembly of NPs at the water-air interface, where a gap between NPs exists in the range of a few $\mathrm{nm}$. Although these organic molecules can be removed with certain procedures, such as plasma etching, the labor and cost post of this treatment process is unfavorable for practical applications of NP assemblies as SERS substrates. To this end, thin layers of $\mathrm{SiO}_{2}$ have also been utilized to stabilize Au NPs after self-assembly at the air-water interface and transfer onto the support material, where the $\mathrm{SiO}_{2}$ layer can be easily removed by chemical etching. More importantly, after removal of the protective layer, the strong Van der Waals attractions drive shortening of interparticle distance to $1-2 \mathrm{~nm}$ nanogaps [43], resulting in an additional $10^{3}-10^{4}$ increase in SERS EF.

It is interesting to note that for isotropic NPs, HCP assembly capable of accommodating the largest amount of NPs to reduce the system energy is the most favorable arrangement. Meanwhile, for anisotropic NPs, the case is much more complicated. For example, bipyramid Au NPs (BNPs) self-assemble into liquid crystalline superstructures with four distinctly oriented packing patterns, including horizontal alignment (H-NLCS), circular arrangement (CNLCS), slanted alignment (S-NLCS), and vertical alignment (VNLCS) (Fig. 3D). However, in terms of strong surface tension and the absence of particle-substrate interactions, only the H-NLCS and V-
NLCS configurations have been followed during the air-water interface assemble process. Moreover, the different packing patterns strongly influence the collective plasmonic coupling properties and, thus, SERS performance. Particularly, the Raman EF of VNLCS and H-NLCS was about 77- and 19-fold greater, respectively, than that for C-NLCS, showing the advantage of using SERS substrate that underwent air-water interface assembly [67].

\subsection{Self-assembly at the liquid-gas-solid three phase interface}

Self-assembly also occurs at the liquid-gas-solid interface when a drop of $\mathrm{Au}$ or $\mathrm{Ag}$ NP solution is transferred onto a solid support. Based on differences in the contact angle and, therefore, surface tension, the drop of solution either remains a hemisphere or spreads across the substrate $[7,56,68]$. With the evaporation of solvent, the NPs form random NP ensembles, coffee rings, or ordered NP assembles at the center of the liquid drop. For example, when a pinned droplet/air/solid line forms, the evaporation of solvent-induced capillary flow drives the transportation of NPs to the rim of the droplet, where a coffee ring-like macroscopic NP assembly forms. Both our group and Xu et al. utilized such nanostructures for sensitive SERS detection of various environmental pollutants $[69,70]$. Optimization of the surface chemistry of Au and $\mathrm{Ag}$ NPs and modification of the wettability of the solid support combined with the effects of Marangoni flow and receding of the contact line enable the homogeneous deposition and formation of concentrated NP assemblies, which are even more effective for SERS analysis than coffee rings [7,25].

\subsection{Evaporation-induced formation of dynamic SERS hotspots}

Compared with the tremendous effect on the design of "fixed" plasmonic hotspots during a dry state, less attention has been paid to SERS hotspots formed in solution. By combining theoretical simulations, in situ synchrotron-radiation small-angle X-ray scattering, dark-field microscopy, and in situ micro-UV, Liu et al. showed as the concentration of NPs in solution increases through evaporation, the electrolyte concentration also increases accordingly. This increased concentration of electrolytes can effectively suppress the electrostatic repulsion between NPs and the Van der Waals attraction, and drive the NPs to approach closer until a new dynamic balance form, where the attractive forces are stronger. These increased attractive forces work as a "trapping well" and immobilize NPs, where the interparticle distance oscillates in a small range as a result of balance between Van der Waals attraction and electrostatic repulsion. Therefore, abundant hotspots in a 3D geometry were dynamically formed. Such a novel and unnoticed matrix not only generates a much-intensified EM field under the excitation of photons, but also provides a structural basis for trapping molecules inside SERS hotspots. Overall, a SERS EF two orders of magnitude or higher than dried substrates was achieved [68].

\section{Gelation based self-assembly of SERS substrate}

\subsection{Halide ion-induced gelation of metal nanowires}

Besides interfacial assembly, for the high surface energy of NCs, many other factors also induce self-assembly. One important example is the halide ion, which is either inventively introduced into the NP system [71] or through metal precursors [27,33,72-74]. Our group developed a series of materials from halide ions, in particular, $\mathrm{Cl}^{-}$-induced deposition of metal $(\mathrm{Au}, \mathrm{Ag}, \mathrm{Cu}, \mathrm{Pd}, \mathrm{Pt}$, Au@Pt, Au@Pd, and Au@Ag@Pd) nanowires (NWs) and their selfassembly into nanoporous film (NPF) (Fig. 4A). The metal nanowires were synthesized using the nonionic surfactant - Triton $\mathrm{X}$ - 


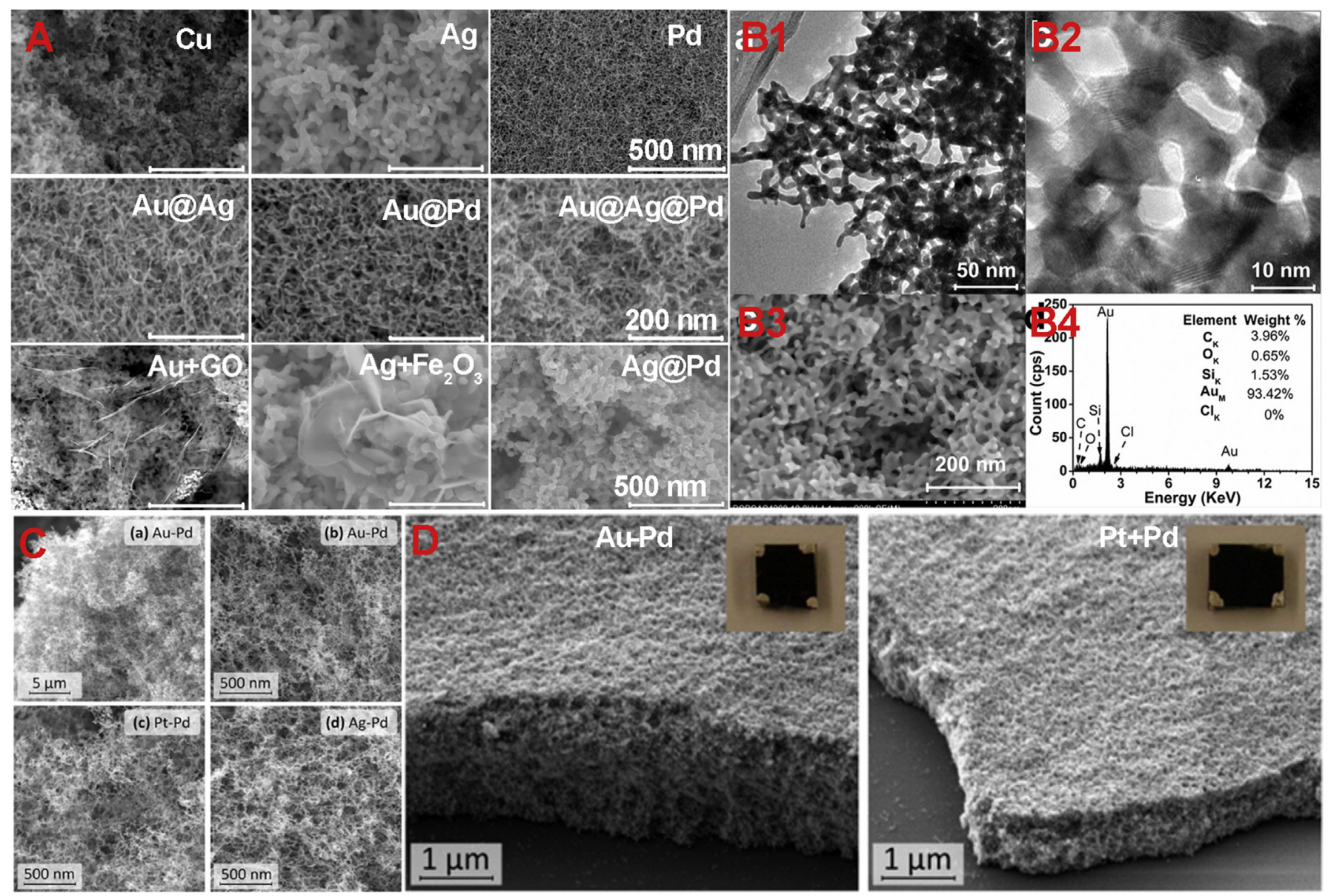

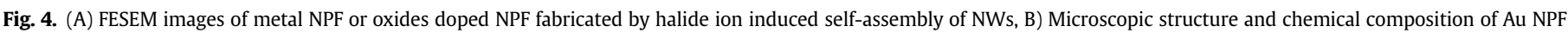

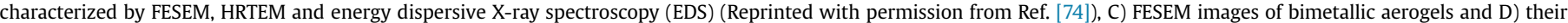
aerogel films deposited on glass substrates (Reprinted with permission from Ref. [82]).

114 (TX-114) as stabilizer and structural director. During the growth of NWs, TX-114 selectively adsorbed the higher energy $\{110\}$ and $\{100\}$ facets of the nascent nanocrystals via weak $\pi-d$ interactions. This induced the NCs to grow into NWs through oriented attachment via the clean $\{111\}$ facets [72]. Since the TX-114 molecules adsorb on the NW surface through relative weak $\pi-\mathrm{d}$ interaction, they can be easily replaced with coexisting ions, making the NWs unstable. Nevertheless, we can remove most of these impurities by cloud point extraction, which also uses TX-114 and makes the synthesized NWs stable for weeks [75]. Otherwise, a uniform NPF forms if the NWs are left undisturbed overnight at $4^{\circ} \mathrm{C}$ [73]. Detailed structural analysis revealed that Au NPF not only contains nanowire junctions, nanopores, and nanowells, but also has abundant nanopores/nanogaps down to $1-2 \mathrm{~nm}$ due to the incomplete fusion of NWs and resulting in a high spatial density of hotspots with Raman EFs up to $10^{9}$ (Fig. 4B). This highly SERS-active film also has the merits of a large area of uniformity, high purity, and being free of background. On the other hand, compared with nanoporous material that fabricated by chemical or electrochemical dealloying, our strategy featured with greenness, low cost and ease of scale-up [76-78]. Using this material, we achieved single molecule SERS detection [74]. We further extended this method to fabrication of Ag NPF that had an even better SERS performance and allowed detection of high toxicity aflatoxins down to $10^{-8} \mathrm{M}$. Moreover, the bottom-up approach used in ( $\mathrm{Au} /$ Ag) NPF fabrication allowed the doping and even distribution of other functional materials into the nanopores, thus conferring them with SERS activity (Fig. 4A) [27,79,80]. The combination of the SERS activity and the functions of the doped materials endows these hybrid SERS substrates with specific functions, which we termed task-specific SERS substrates. As a proof-of-concept, we coprecipitated nanosorbent $\left(\gamma-\mathrm{Fe}_{2} \mathrm{O}_{3}\right.$ nanosheets, a good sorbent for inorganic Arsenic ions) and nanocatalyst (Pd overlayer/branches) into Ag NPFs, and successfully performed ultra-sensitive SERS detection of arsenic ions in the presence of a high concentration of coexisting ions and monitored the catalyzed reactions with SERS with a millisecond time resolution [27,33].

\subsection{Noble metal aerogels from gelation of nanoparticles}

Another group of noble metal self-assembly with potential application as SERS substrate is aerogel or hydrogel. Just like nanoporous film, these materials have abundant nanopores that may work as 3D SERS hotspots. Eychmuller's group first reported the self-assemble of nanoparticles into non-supported monometallic ( $\mathrm{Au}, \mathrm{Ag}, \mathrm{Pt}$ ) and bimetallic ( $\mathrm{Au} / \mathrm{Ag}$ and $\mathrm{Pt} / \mathrm{Ag}$ ) aerogels without chemical cross-linkers (Fig. 4C) [81,82]. This was fulfilled through the interconnection of concentrated citrate stabilized nanoparticles. Coincidentally, the aerogel formation process is also via the nanowire intermediates, or the nanoparticles first growth into nanowires and then 3D monoliths. The crucial step for the destabilizing of these NPs is the stepwise concentration the synthesized NPs by rotary evaporation or ultracentrifugation to $10^{-6} \mathrm{M}$ NP or above. The concentrated NPs gelates into aerogel after a long 
time aging process of c.a., 2-4 weeks. Although in presence of destabilizer like $\mathrm{H}_{2} \mathrm{O}_{2}$ or ethanol, the process can be accelerated to some extent ( 10 days). Obviously the extended aging time is the main bottleneck for the practical application of this novel material in the analytic community. Meanwhile, the high concentration of NPs means the necessary of the time and labor consuming preconcentration process, as well as the high risk of forming undesired NP aggregates. To this end, the same group also developed a dopamine-induced assembly of beta-cyclodextrin modified Au NPs to form Au aerogel (Fig. 4D). Therein, the dopamine triggers the interconnection of NPs either by forming host-guest inclusion complex with $\beta-\mathrm{CD}$, or directly adsorbs and interacts with the $\mathrm{Au}$ surface through the strong affinity between dopamine and the $\mathrm{Au}$ core [83]. The gelation process was further shortened to $6 \mathrm{~h}$ or lower and adopted to synthesis metal alloy aerogel by reduction the precursors at $60^{\circ} \mathrm{C}$ and keep the result dispersion at the same temperature [84].

Currently, the report on the analytic application of those aerogel is as electrochemical sensor for small molecule like ethanol [83], which is benefited from their large surface area, abundant active sites, and high porosity based high electrocatalytic activity [84]. However, considering their pronounced surface plasmon resonances related specific optical properties, there is no double that aerogels from metal nanoparticles may also find future applications as advanced optical sensors and ultrasensitive detectors [85].

\section{Taking full advantage of the assembly process: integration of target analyte recognition and capture capacity into the assembly}

\subsection{Coupling molecular recognition with self-assembly}

\subsubsection{Molecular recognition with biomolecules}

The ability to precisely recognize biomolecules, including DNA, peptides/proteins, and enzymes, makes these materials the basis for all life events. This has been repurposed to design 3D structures of various materials and functionalities with nanoscale accuracy and, thereby, facilitate SERS analysis of a variety of analytes, ranging from inorganic ions to biological events, with high sensitivity and precision. The most successful applications of biomoleculetriggered molecular recognition is in SERS immunoassays [86], where it enables the SERS tag to be captured and self-assembled on various analyte surfaces [87] and facilitates the formation of highly SERS-active nanoarchitectures. This strategy has been successfully used to design a SERS sensor with a high sensitive and the ability to quantitatively detect intracellular telomerase [88]. Taking advantage of telomerase triggering of telomerase primer (TP) strand extension with repeat units (TTAGGG) using intrinsic RNAs as templates after activation by TP, an intracellular telomerase SERS sensor was designed. In this sensor, four gold NPs were interconnected into pyramids using single-stranded DNA complementary to the cyanine 5 (Cy5) modified reporter sequence (RS) and telomerase primer (TP). After recognizing TP, the telomerase initiates the extension of the TP strand with repeat units and replaces the Cy5-embedded RS strand, thus turning off the SERS signal (Fig. 5A and B).

\subsubsection{Hydrogen bonding}

For specific detection of $\mathrm{HClO}$, our group designed a coresatellite nanostructure with a high density of hotspots with good SERS performance. In this sensor, the core gold NPs (AuNPs, $\sim 100 \mathrm{~nm}$ ) with a monolayer of 4-mercaptoimidazole (MI) ligands were covalently linked to a thiol-derived Si wafer (MI-AuNPs@SH$\mathrm{Si})$, and multiple satellite AuNPs ( $12 \mathrm{~nm})$ decorated with both MI and a Raman reporter assembled around the core MI-AuNPs via intermolecular $\mathrm{NH} \cdots \mathrm{N}$ hydrogen bonds (HBs) provided by the neighboring imidazole moiety. In the presence of $\mathrm{HClO}$, the imidazole moieties were quickly oxidized, resulting in the destruction of HBs and, thus, separating the satellite AuNPs from the core AuNPs and turning off the SERS signal. This HB-induced assembly/disassembly of Au clusters is highly specific for $\mathrm{HClO}$ over other several reactive oxygen/nitrogen species with a limit of detection down to $1.2 \mu \mathrm{mol} \mathrm{L}^{-1}$ and can be used in analysis of water and human serum samples with satisfactory results (Fig. 5C) [31].

\subsubsection{Host-guest chemistry}

In addition to biomolecule and hydrogen bond-mediated molecular recognition, host-guest chemistry also has an effect on the capture of target molecules. One important example of this is the utilization of cucurbit[n]urils ( $\mathrm{CB}[\mathrm{n}])$ as hosts for hydrophobic and/ or small cationic molecules and internal standards for quantification during SERS analysis. After bridging adjacent Au NPs through two electronegative carbonyl portals, gold colloids self-assemble into a convenient platform in a controllable and reproducible manner. With this platform, a SERS-based method has been developed for SERS detection of hydrophobic nonfluorescent molecules at low concentrations in aqueous solutions. This largely simplifies sample preparation and improves the limits of detection of polycyclic aromatic hydrocarbons by at least 3 orders of magnitude $\left(10^{-11} \mathrm{M}\right)$ over existing SERS methods (Fig. 5D) [29].

\subsection{Simultaneous self-assembly of SERS substrates and enrichment of target analyte}

Although SERS hotspots have been claimed to have single molecule detection sensitivities, precise delivery of analytes to these sites is still a long-standing challenge for SERS analysis and hinders the detection of single molecules of SERS-active analytes in very dilute solutions. Compared with the capillary force-driven diffusion of analytes, co-evaporation of analyte and SERS-active NPs in confined regions has a higher probability of localizing the target analyte into SERS hotspots, as well as integrates the generation of SERS substrates and enrichment of analytes into one step. Ag NPs and analytes have been reported to be concentrated on a slippery liquid-infused porous superhydrophobic surface in both aqueous and organic phases and are detectable by SERS at a molar level of analyte [7]. In addition to having the lowest limit of detection for any SERS-based detection reported thus far, this method can detect analytes present in liquid, solid, and air phases that can be extracted using a suitable liquid solvent and subsequently detected. On the other hand, the coffee ring effect has been reported to be an effective pre-concentration procedure for ultrasensitive drop-coating deposition Raman analysis of microcystin-LR [89]. Coupled with SERS enhancement through construction of SERS substrates and pre-concentration of analytes, the sensitivity can be largely enhanced. For example, based on differences in surface tension induced by analyte, the coffee ring effect alone can enrich the analyte by a factor ranging from 350 to 850 [70]. Combined with a SERS EF of $10^{6}$ to $10^{7}$, the analytic EF can reach $10^{8}$ or more, and is sufficient for SM-SERS of most probe molecules.

\section{Assembly makes perfect: new opportunities created by large self-assembling SERS substrates}

\subsection{Large uniform bi-functional SERS substrates for the study of surface events}

One important advantage of self-assembled SERS substrates is their large area of uniformity, which not only enables quantitative 

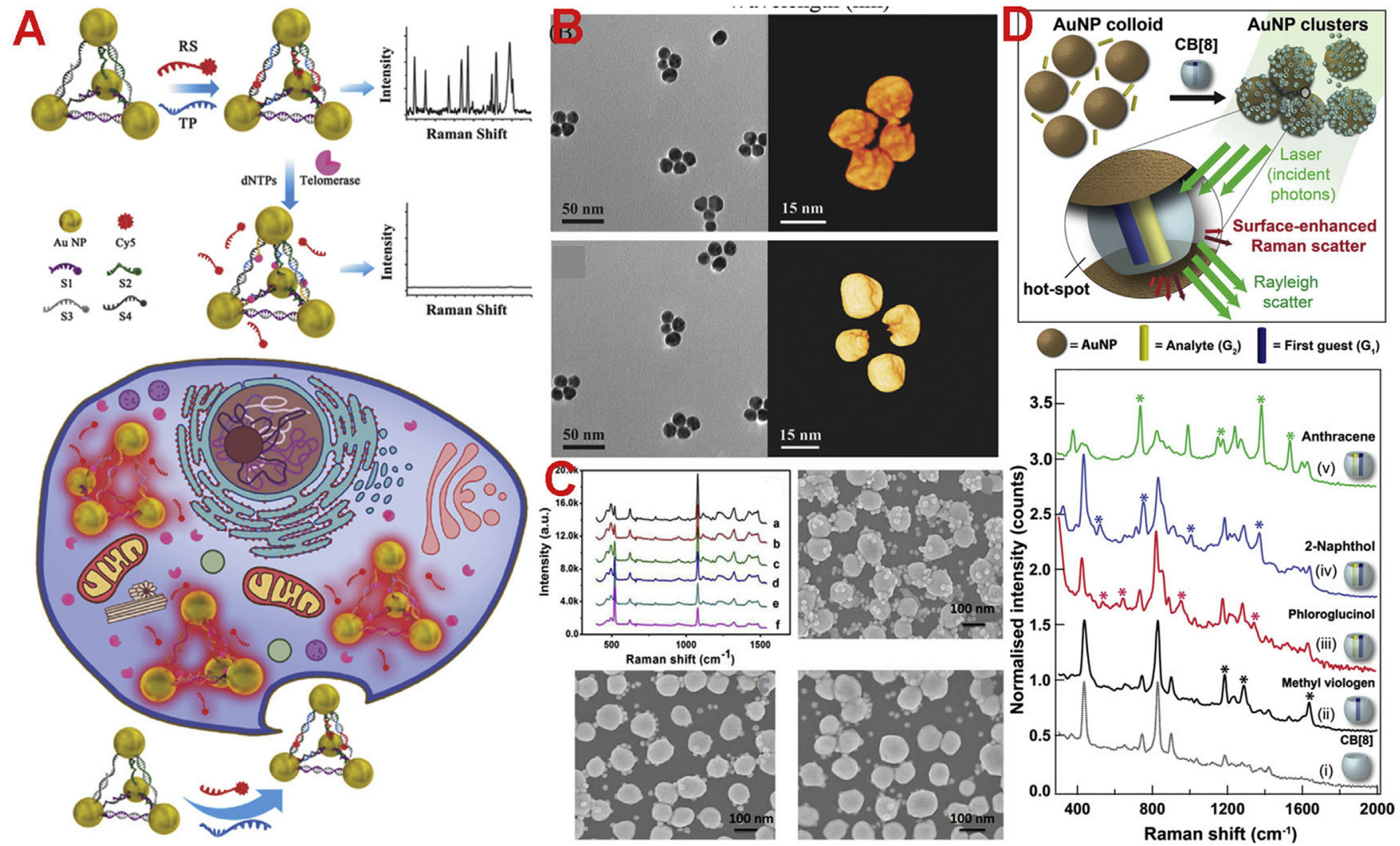

Fig. 5. A) Schematic illustration of intracellular of telomerase activity sensor based on Au NPs pyramid, and B) the TEM and 3D reconfiguration electron-tomography showed the good stability of the sensor (Reprinted with permission from Ref. [88]), C) SERS spectra and FESEM images of core-satellite HClO sensor in the presence of target analyte with different concentration (Reprinted with permission from Ref. [31]). D) Schematic of CB [8] induced aggregates of Au NP, and localizes the analyte inside SERS hotspot by host-guest chemistry, and the SERS of the CB [8] complexed with different guest molecules (Reprinted with permission from Ref. [29]).

SERS analysis [90], but also in situ SERS studies of surface events, e.g. the fate of surface plasmon resonance (SPR)-generated hot electrons (HEs) [80]. Despite its demonstrated capacity for in situ SERS surging of catalyzed reactions for kinetic and mechanistic studies, continuous excitation of the same area with a high-power density $\left(10^{7} \mathrm{~mW} \mathrm{~cm}^{-2}\right.$ or above) laser can generate SPR-generated HEs and photothermal (PT) effects as byproducts that may influence reaction kinetics or other pathways under dark conditions. This may result in in situ SERS signals being not as virgin as expected. To this end, we compared the kinetics of p-NTP reduction reactions under illumination with a laser at different wavelengths and power densities. Moreover, took advantage of the large area uniformity of Au@Pd and Ag@Pd nanoporous films, we performed Raman mapping or line scanning at $\mathrm{mm}^{2}$ or $\mathrm{cm}$ with a stepsize larger than the spotsize of the laser to prevent the light from affecting the reaction kinetics (Fig. 6A). A linear dependence was observed between the power density and rate constant of the pNTP reaction on the Au@Pd film, which is a direct result of SPRgenerated HE-induced reaction acceleration. Based on this unexpected discovery, we proposed an in situ SERS method to track the fate of SPR-generated HEs. With this method, Ag pinholes were identified as a key factor for leakage of HEs into the surrounding ions/molecules $[27,79,80]$, including $O$ species like lattice $O$ and surface adsorbed molecular $\mathrm{O}_{2}$, which sheds light onto why Ag is seldom employed as a plasmonic co-catalyst and provides a new viewpoint by which to design highly efficient light-utilizing plasmonic nanocatalysts.

Of note is if we in-situ monitoring the chemical reaction catalyzed by SERS and catalytic bi-function NPs in solution, c.a.,
Ag@Pt nanocube [91,92] or Ag@SiO $@$ Au NPs [93], for the continuous Brown movement of NPs, the light effect was largely suppressed or averaged. Indeed, the initial observation of SPR facilitated activation of $\mathrm{O}_{2}$ on $\mathrm{Au}$ and $\mathrm{Ag}$ surface was also performed by Au or Ag NPs supported on Si substrate [94]. This, again demonstrate the advantage of large area SERS substrate in surface process study.

\subsection{Recyclable SERS substrate using combinations of different materials}

One long-standing drawback of traditional SERS substrates is that they can be used only once, as even trace amounts of residual analyte can influence the SERS substrate. This also hinders the use of SERS in real-time online detection of organic pollutants under continuous flow. The introduction of a photocatalyst, such as $\mathrm{TiO}_{2}$, and the catalytic removal of surface-adsorbed analyte using UV light is a plausible solution. Moreover, $\mathrm{TiO}_{2}$ with a well-defined morphology, such as that used in nanotube arrays, is an ideal scaffold for the self-assembly of SERS active Au nanostructures. Using this novel strategy, a composite substrate integrating SERS, Au NP activity, and the self-cleaning capacity of $\mathrm{TiO}_{2}$ was designed [95]. After analysis by SERS, the adsorbed dyes and environmental pollutants were completely mineralized into small molecules, including water and $\mathrm{CO}_{2}$, and the substrate was recycled and reused at least three times without loss of SERS activity. This indicates these are promising SERS platforms for the detection of multiple different molecular species. This material may also be applicable in in situ SERS studies of photocatalysis. 

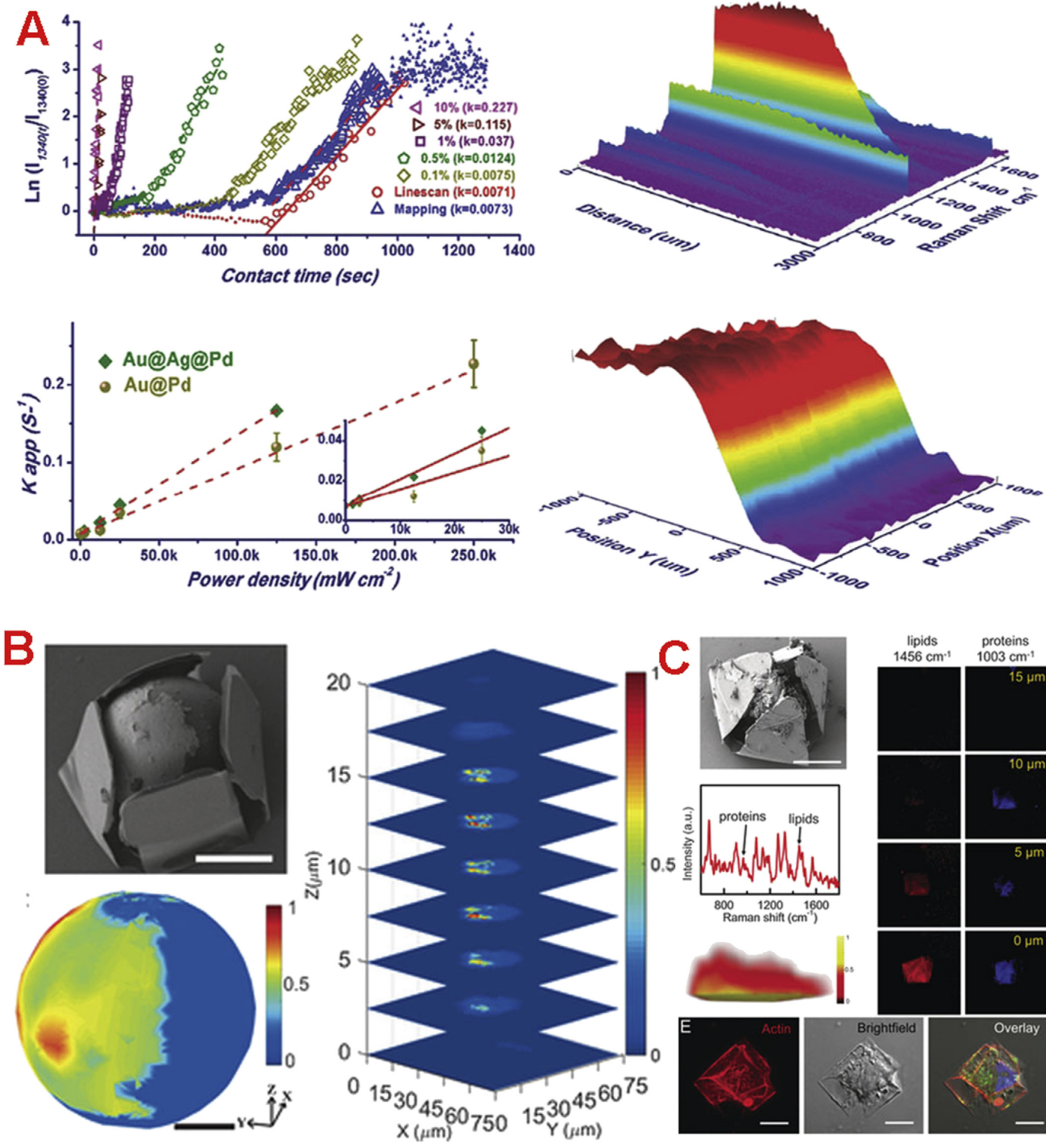

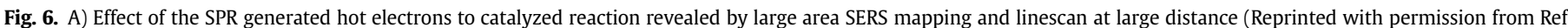

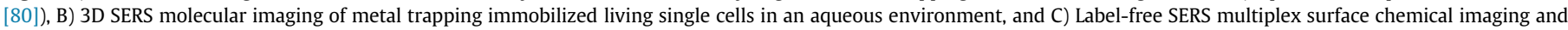
molecular profiling of single live cells encapsulated within MTs (Reprinted with permission from Ref. [34]).

\subsection{Macroscopic plasmonic assembly for SERS analysis of single living cells}

Compared to analytes in solution or on the surface of different shaped objectives that can be SERS analyzed using traditional SERS substrate and plaster [46,61], SERS measurement of 3D cell surface molecular expression on living cells is very challenging. This is partly due to the lack of platforms with the multiple-functions of cell capture and multiplexed, nonperturbative SERS analysis. This is achieved by coating gold nanostar self-assemblies modified with patterned bilayer mechanical traps (MTs). The MTs capture cells and release energy from differential residual stress. Moreover, the MTs enable close contact between the SERS active Au nanostars and the $3 \mathrm{D}$ cell surfaces, making it feasible to spectroscopically analyze 
biological events with the molecular specificity of Raman spectroscopy. This offers a direct, quantitative, label-free method of chemical analysis in a nondestructive and real-time manner with subcellular microscale spatial resolution (Fig. 6B and C). Upon becoming trapped in the GNS-modified MTs and then being kept under standard incubator conditions, the cells remained viable for up to at least two days. Using this platform, it is very easy to measure the SERS bands from protein and lipid components of the cell membrane. Furthermore, by overlaying the optical and SERS images, a spatial map of the different molecular distributions within the cell membrane can also be obtained [34]. This neoteric cell capture and SERS enhancement platform will lead to the manipulation and long-term molecular mapping of the cell membrane in different milieu at a single-cell level.

\section{Perspectives and outlook}

SERS substrates have a vital role in high performance SERS analysis, and the importance of this technology in studies on various biological and physico-chemical processes makes the design and fabrication of SERS substrates a key research topic in the field of nanotechnology. The self-assembly of NPs stimulated by the various factors discussed in this review provides an effective and elegant solution for fabrication of hierarchical nanoarchitectures with abundant and evenly distributed SERS hotspots. However, the unparalleled SM sensitivity and molecular specificity of SERS is, in most cases, due to probe molecules or other chemicals with simple structures and large Raman cross-sections. This means that, besides designing and fabricating SERS hotspots with large EFs, more attention should be paid to how to precisely position the analyte inside these hotspots. To this end, using macromolecules with a capacity for guest-host chemistry molecule recognition as a glue to induce self-assembly [29] or building large areas of hot zones to balance SERS EF and analyte accessibility are possible solutions [90]. Meanwhile, the magnitude and spatial distribution of SERS hotspots are largely based on interparticle gap distances, which are in a highly dynamic state both in solutions and on solid assemblies [22]. Therefore, understanding and taking advantage of these highly dynamic SERS hotspots are very important, and may create new opportunities for designing new generations of SERS substrates [68]. Moreover, better application of SERS in in situ and in vivo studies relies on better integration of the catalytic and cellular activity components into the plasmonic assembly and designing highly task-specific SERS substrates [33]. Currently, this is satisfied using direct coating [96] or mechanical trapping [33,34], and there is no doubt that more effective and straightforward tactics are still in demand.

\section{Acknowledgments}

The authors acknowledge the anonymous reviewers for their constructive comments and suggestions. This work was financially supported by the National Key R\&D Program of China (2016YFA0203102), and the National Natural Science Foundation of China (21577157). Liu R. acknowledges support from the Youth Innovation Promotion Association of Chinese Academy of Sciences.

\section{References}

[1] D.L. Jeanmaire, R.P. Vanduyne, J. Electroanal. Chem. 84 (1977) 1-20.

[2] M. Fleischmann, P.J. Hendra, A.J. McQuillan, Chem. Phys. Lett. 26 (1974) $163-166$.

[3] M.G. Albrecht, J.A. Creighton, J. Am. Chem. Soc. 99 (1977) 5215-5217.

[4] A.M. Michaels, M. Nirmal, L.E. Brus, J. Am. Chem. Soc. 121 (1999) 9932-9939.

[5] S. Nie, S.R. Emory, Science 275 (1997) 1102-1106.

[6] K. Kneipp, Y. Wang, H. Kneipp, L.T. Perelman, I. Itzkan, R.R. Dasari, M.S. Feld, Phys. Rev. Lett. 78 (1997) 1667-1670.
[7] S. Yang, X. Dai, B.B. Stogin, T.S. Wong, Proc. Natl. Acad. Sci. U. S. A. 113 (2016) $268-273$.

[8] S.Y. Ding, J. Yi, J.F. Li, B. Ren, D.Y. Wu, R. Panneerselvam, Z.Q. Tian, Nat. Rev. Mater. 1 (2016) 16021.

[9] A.B. Zrimsek, N. Chiang, M. Mattei, S. Zaleski, M.O. McAnally, C.T. Chapman, A.I. Henry, G.C. Schatz, R.P. Van Duyne, Chem. Rev. 117 (2017) 7583-7613.

[10] L.A. Austin, B. Kang, M.A. El-Sayed, Nano Today 10 (2015) 542-558.

[11] P.G. Etchegoin, E.C. Le Ru, Phys. Chem. Chem. Phys. 10 (2008) 6079-6089.

[12] G. Chen, Y. Wang, M.X. Yang, J. Xu, S.J. Goh, M. Pan, H.Y. Chen, J. Am. Chem. Soc. 132 (2010) 3644-3645.

[13] L. Scarabelli, M. Coronado-Puchau, J.J. Giner-Casares, J. Langer, L.M. Liz-Marzan, ACS Nano 8 (2014) 5833-5842.

[14] N. Pazos-Perez, C.S. Wagner, J.M. Romo-Herrera, L.M. Liz-Marzan, F.J. Garcia de Abajo, A. Wittemann, A. Fery, R.A. Alvarez-Puebla, Angew. Chem. Int. Ed. 51 (2012) 12688-12693.

[15] P.H.C. Camargo, M. Rycenga, L. Au, Y.N. Xia, Angew. Chem. Int. Ed. 48 (2009) 2180-2184.

[16] Y. Li, J.D. Cain, E.D. Hanson, A.A. Murthy, S.Q. Hao, F.Y. Shi, Q.Q. Li, C. Wolverton, X.Q. Chen, V.P. Dravid, Nano Lett. 16 (2016) 7696-7702.

[17] Y. Fang, N.H. Seong, D.D. Dlott, Science 321 (2008) 388-392.

[18] M. Chirumamilla, A. Toma, A. Gopalakrishnan, G. Das, R.P. Zaccaria, R. Krahne, E. Rondanina, M. Leoncini, C. Liberale, F. De Angelis, E. Di Fabrizio, Adv. Mater. 26 (2014) 2353-2358.

[19] E.J. Smythe, M.D. Dickey, J.M. Bao, G.M. Whitesides, F. Capasso, Nano Lett. 9 (2009) 1132-1138.

[20] A. Klinkova, R.M. Choueiri, E. Kumacheva, Chem. Soc. Rev. 43 (2014) 3976-3991.

[21] P.Y. Kim, J.W. Oh, J.M. Nam, J. Am. Chem. Soc. 137 (2015) 8030-8033.

[22] A. Lee, G.F.S. Andrade, A. Ahmed, M.L. Souza, N. Coombs, E. Tumarkin, K. Liu, R. Gordon, A.G. Brolo, E. Kumacheva, J. Am. Chem. Soc. 133 (2011) 7563-7570.

[23] X.S. Shen, L.Y. Chen, D.H. Li, L.F. Zhu, H. Wang, C.C. Liu, Y. Wang, Q.H. Xiong, H.Y. Chen, ACS Nano 5 (2011) 8426-8433.

[24] C. Zhu, G. Meng, P. Zheng, Q. Huang, Z. Li, X. Hu, X. Wang, Z. Huang, F. Li, N. Wu, Adv. Mater. 28 (2016) 4871-4876.

[25] P. Li, Y. Li, Z.K. Zhou, S. Tang, X.F. Yu, S. Xiao, Z. Wu, Q. Xiao, Y. Zhao, H. Wang, P.K. Chu, Adv. Mater. 28 (2016) 2511-2517.

[26] C.Y. Niu, B.F. Zou, Y.Q. Wang, L. Cheng, H.H. Zheng, S.M. Zhou, Langmuir 32 (2016) 858-863.

[27] R. Liu, J.F. Liu, Z.M. Zhang, L.Q. Zhang, J.F. Sun, M.T. Sun, G.B. Jiang, J. Phys. Chem. Lett. 5 (2014) 969-975.

[28] Q.H. Guo, M.M. Xu, Y.X. Yuan, R.N. Gu, J.L. Yao, Langmuir 32 (2016) 4530-4537.

[29] S. Kasera, F. Biedermann, J.J. Baumberg, O.A. Scherman, S. Mahajan, Nano Lett. 12 (2012) 5924-5928.

[30] S. Li, L.G. Xu, W. Ma, H. Kuang, L.B. Wang, C.L. Xu, Small 11 (2015) 3435-3439.

[31] J.F. Sun, R. Liu, J.J. Tang, Z.M. Zhang, X.X. Zhou, J.F. Liu, ACS Appl. Mater. Interfaces 7 (2015) 16730-16737.

[32] W. Ren, Y. Fang, E. Wang, ACS Nano 5 (2011) 6425-6433.

[33] R. Liu, J.F. Sun, D. Cao, L.Q. Zhang, J.F. Liu, G.B. Jiang, Chem. Commun. 51 (2015) 1309-1312.

[34] Q. Jin, M. Li, B. Polat, S.K. Paidi, A. Dai, A. Zhang, J.V. Pagaduan, I. Barman, D.H. Gracias, Angew. Chem. Int. Ed. 56 (2017) 3822-3826.

[35] L.C. Brousseau, J.P. Novak, S.M. Marinakos, D.L. Feldheim, Adv. Mater. 11 (1999) 447-449.

[36] L. Guerrini, Z. Krpetic, D. van Lierop, R.A. Alvarez-Puebla, D. Graham, Angew. Chem. Int. Ed. 54 (2015) 1144-1148.

[37] L.J. Xu, Z.C. Lei, J.X. Li, C. Zong, C.J. Yang, B. Ren, J. Am. Chem. Soc. 137 (2015) 5149-5154.

[38] D. van Lierop, Z. Krpetic, L. Guerrini, I.A. Larmour, J.A. Dougan, K. Faulds, D. Graham, Chem. Commun. 48 (2012) 8192-8194.

[39] V.T. Cong, E.O. Ganbold, J.K. Saha, J. Jang, J. Min, J. Choo, S. Kim, N.W. Song, S.J. Son, S.B. Lee, S.W. Joo, J. Am. Chem. Soc. 136 (2014) 3833-3841.

[40] H. Jia, X.T. Bai, L.Q. Zheng, CrystEngcomm 14 (2012) 2920-2925.

[41] L. Zhao, T.H. Kim, H.W. Kim, J.C. Ahn, S.Y. Kim, Acta Biomater. 20 (2015) $155-164$.

[42] D. Bekana, R. Liu, M. Amde, J.F. Liu, ACS Appl. Mater. Interfaces 9 (2017) 513-520.

[43] Y. Shin, J.W. Song, D.C. Kim, T. Kang, Adv. Mater. 27 (2015) 4344-4350.

[44] G. Lu, H. Li, C. Liusman, Z.Y. Yin, S.X. Wu, H. Zhang, Chem. Sci. 2 (2011) 1817-1821.

[45] X. Ling, L.M. Xie, Y. Fang, H. Xu, H.L. Zhang, J. Kong, M.S. Dresselhaus, J. Zhang, Z.F. Liu, Nano Lett. 10 (2010) 553-561.

[46] W.G. Xu, X. Ling, J.Q. Xiao, M.S. Dresselhaus, J. Kong, H.X. Xu, Z.F. Liu, J. Zhang, Proc. Natl. Acad. Sci. U. S. A. 109 (2012) 9281-9286.

[47] J.P. Lu, J.H. Lu, H.W. Liu, B. Liu, L.L. Gong, E.S. Tok, K.P. Loh, C.H. Sow, Small 11 (2015) 1792-1800.

[48] G. Kim, M.S. Kim, C.H. Hyun, S.M. Hong, K.Y. Ma, H.S. Shin, H. Lim, ACS Nano 10 (2016) 11156-11162.

[49] Q. Liu, J.B. Shi, J.T. Sun, T. Wang, L.X. Zeng, G.B. Jiang, Angew. Chem. Int. Ed. 50 (2011) 5913-5917.

[50] W.G. Xu, J.Q. Xiao, Y.F. Chen, Y.B. Chen, X. Ling, J. Zhang, Adv. Mater. 25 (2013) 928-933.

[51] X.H. Li, W.C.H. Choy, X.G. Ren, D. Zhang, H.F. Lu, Adv. Funct. Mater. 24 (2014) 3114-3122. 
[52] R. Xiong, K.S. Hu, S.D. Zhang, C.H. Lu, V.V. Tsukruk, ACS Nano 10 (2016) 6702-6715.

[53] J. Zhao, M. Sun, Z. Liu, B. Quan, C. Gu, J. Li, Sci. Rep. 5 (2015) 16019.

[54] X. Yang, H. Yu, X. Guo, Q. Ding, T. Pullerits, R. Wang, G. Zhang, W. Liang, M. Sun, Mater. Today Energy 5 (2017) 72-78.

[55] Z.G. Dai, X.H. Xiao, W. Wu, Y.P. Zhang, L. Liao, S.S. Guo, J.J. Ying, C.X. Shan, M.T. Sun, C.Z. Jiang, Light Sci. Appl. 4 (2015) e342.

[56] S. Lee, M.G. Hahm, R. Vajtai, D.P. Hashim, T. Thurakitseree, A.C. Chipara, P.M. Ajayan, J.H. Hafner, Adv. Mater. 24 (2012) 5261-5266.

[57] N. Shah, F.P. Zamborini, ACS Nano 9 (2015) 10278-10286.

[58] X. Guo, Y. Yin, Z. Tan, Z. Zhang, Y. Chen, J. Liu, Environ. Sci. Technol. Lett. 3 (2016) 381-385.

[59] Y.K. Xu, M.P. Konrad, W.W.Y. Lee, Z.W. Ye, S.E.J. Bell, Nano Lett. 16 (2016) $5255-5260$

[60] J.W. Liu, S.Y. Zhang, H. Qi, W.C. Wen, S.H. Yu, Small 8 (2012) 2412-2420.

[61] L.B. Zhong, J. Yin, Y.M. Zheng, Q. Liu, X.X. Cheng, F.H. Luo, Anal. Chem. 86 (2014) 6262-6267.

[62] Y. Ma, H. Liu, M. Mao, J. Meng, L. Yang, J. Liu, Anal. Chem. 88 (2016) $8145-8151$

[63] K. Kim, H.S. Han, I. Choi, C. Lee, S. Hong, S.H. Suh, L.P. Lee, T. Kang, Nat Commun. 4 (2013) 2182.

[64] S. Acharya, J.P. Hill, K. Ariga, Adv. Mater. 21 (2009) 2959-2981.

[65] A.R. Tao, J.X. Huang, P.D. Yang, Acc. Chem. Res. 41 (2008) 1662-1673.

[66] A. Tao, F. Kim, C. Hess, J. Goldberger, R.R. He, Y.G. Sun, Y.N. Xia, P.D. Yang, Nano Lett. 3 (2003) 1229-1233.

[67] Q. Shi, K.J. Si, D. Sikdar, L.W. Yap, M. Premaratne, W. Cheng, ACS Nano 10 (2016) 967-976

[68] H.L. Liu, Z.L. Yang, L.Y. Meng, Y.D. Sun, J. Wang, L.B. Yang, J.H. Liu, Z.Q. Tian, J. Am. Chem. Soc. 136 (2014) 5332-5341.

[69] J.W. Xu, J.J. Du, C.Y. Jing, Y.L. Zhang, J.L. Cui, ACS Appl. Mater. Interfaces 6 (2014) 6891-6897.

[70] W. Wang, Y. Yin, Z. Tan, J. Liu, Nanoscale 6 (2014) 9588-9593.

[71] Z. Zhang, H. Li, F. Zhang, Y. Wu, Z. Guo, L. Zhou, J. Li, Langmuir 30 (2014) $2648-2659$.

[72] R. Liu, J.F. Liu, G.B. Jiang, Chem. Commun. 46 (2010) 7010-7012.

[73] R. Liu, J.F. Liu, S.J. Yu, Q. Liu, G.B. Jiang, Chem. Commun. 47 (2011) 1613-1615.

[74] R. Liu, J.F. Liu, X.X. Zhou, M.T. Sun, G.B. Jiang, Anal. Chem. 83 (2011) 9131-9137.
[75] J.F. Liu, R. Liu, Y.G. Yin, G.B. Jiang, Chem. Commun. (2009) 1514-1516.

[76] J. Erlebacher, M.J. Aziz, A. Karma, N. Dimitrov, K. Sieradzki, Nature 410 (2001) 450-453.

[77] P. Liu, P.F. Guan, A. Hirata, L. Zhang, L.Y. Chen, Y.R. Wen, Y. Ding, T. Fujita J. Erlebacher, M.W. Chen, Adv. Mater. 28 (2016) 1753-1759.

[78] L.H. Qian, X.Q. Yan, T. Fujita, A. Inoue, M.W. Chen, Appl. Phys. Lett. (2007) 90.

[79] R. Liu, L.Q. Zhang, C. Yu, M.T. Sun, J.F. Liu, G.B. Jiang, Adv. Mater. 29 (2017), 1604571.

[80] R. Liu, Z. He, J. Sun, J. Liu, G. Jiang, Small 12 (2016) 6378-6387.

[81] N.C. Bigall, A.K. Herrmann, M. Vogel, M. Rose, P. Simon, W. Carrillo-Cabrera, D. Dorfs, S. Kaskel, N. Gaponik, A. Eychmuller, Angew. Chem. Int. Ed. 48 (2009) 9731-9734.

[82] A.K. Herrmann, P. Formanek, L. Borchardt, M. Klose, L. Giebeler, J. Eckert, S. Kaskel, N. Gaponik, A. Eychmüller, Chem. Mater. 26 (2014) 1074-1083.

[83] D. Wen, W. Liu, D. Haubold, C. Zhu, M. Oschatz, M. Holzschuh, A. Wolf, F. Simon, S. Kaskel, A. Eychmuller, ACS Nano 10 (2016) 2559-2567.

[84] C. Zhu, Q. Shi, S. Fu, J. Song, H. Xia, D. Du, Y. Lin, Adv. Mater. 28 (2016) 8779-8783.

[85] N. Gaponik, A.K. Herrmann, A. Eychmuller, J. Phys. Chem. Lett. 3 (2012) 8-17.

[86] Z.Y. Wang, S.F. Zong, L. Wu, D. Zhu, Y.P. Cui, Chem. Rev, 117 (2017) 7910-7963.

[87] Y.Q. Wang, B. Yan, L.X. Chen, Chem. Rev. 113 (2013) 1391-1428.

[88] L.G. Xu, S. Zhao, W. Ma, X.L. Wu, S. Li, H. Kuang, L.B. Wang, C.L. Xu, Adv. Funct. Mater. 26 (2016) 1602-1608.

[89] R.A. Halvorson, P.J. Vikesland, Environ. Sci. Technol. 45 (2011) 5644-5651.

[90] H.Y. Chen, M.H. Lin, C.Y. Wang, Y.M. Chang, S. Gwo, J. Am. Chem. Soc. 137 (2015) 13698-13705.

[91] Y. Zhang, J. Liu, J. Ahn, T.H. Xiao, Z.Y. Li, D. Qin, ACS Nano 11 (2017) 5080-5086.

[92] Y. Wu, X. Sun, Y. Yang, J. Li, Y. Zhang, D. Qin, Acc. Chem. Res. 50 (2017) 1774-1784.

[93] Y.R. Wu, D. Su, D. Qin, ChemNanoMat 3 (2017) 245-251.

[94] Y.F. Huang, M. Zhang, L.B. Zhao, J.M. Feng, D.Y. Wu, B. Ren, Z.Q. Tian, Angew Chem. Int. Ed. 53 (2014) 2353-2357.

[95] X.H. Li, G.Y. Chen, L.B. Yang, Z. Jin, J.H. Liu, Adv. Funct. Mater. 20 (2010) 2815-2824.

[96] J.F. Li, Y.J. Zhang, S.Y. Ding, R. Panneerselvam, Z.Q. Tian, Chem. Rev. 117 (2017) 5002-5069. 